\title{
ER Dynamics and Derangement in Neurological Diseases
}

\author{
Tomoyuki Yamanaka* and Nobuyuki Nukina \\ Laboratory of Structural Neuropathology, Graduate School of Brain Science, Doshisha University, Kyoto, Japan
}

The endoplasmic reticulum (ER) is a morphologically dynamic organelle containing different membrane subdomains with distinct cellular functions. Numerous observations have revealed that ER stress response induced by disturbed ER homeostasis is linked to various neurological/neurodegenerative disorders. In contrast, recent findings unveil that ER structural derangements are linked to the progression of several neurological diseases. The derangements involve two distinct, and likely opposing pathways. One is dysfunction of ER dynamics machinery, leading to disruption of ER network organization. Another one is facilitation of pre-existing machinery, leading to generation of markedlyordered de novo membranous structure. Restoring the ER network can be the effective way toward the cure of ER-deranged neurological disorders.

Keywords: endoplasmic reticulum, ER architecture, ER dynamics, neurological disease, neurodegeneration, NF-Y

\section{OPEN ACCESS}

Edited by:

Gen Sobue,

Nagoya University, Japan

Reviewed by:

Corinne Lasmezas,

Scripps Florida, United States

Smitha Karunakaran,

Indian Institute of Science, India

*Correspondence:

Tomoyuki Yamanaka toyamana@mail.doshisha.ac.jp

Specialty section:

This article was submitted to

Neurodegeneration,

a section of the journal

Frontiers in Neuroscience

Received: 26 September 2017 Accepted: 05 February 2018

Published: 20 February 2018

Citation:

Yamanaka T and Nukina N (2018) ER

Dynamics and Derangement in

Neurological Diseases.

Front. Neurosci. 12:91.

doi: 10.3389/fnins.2018.00091

\section{INTRODUCTION}

Endoplasmic reticulum (ER) is a continuous membrane organelle dispersing throughout the cells. It consist of two differentially shaped membranous domains; the nuclear envelope, a highly regulated membrane barrier that separates the nucleus from the cytoplasm, and the peripheral ERs including ribosome-studded rough ER (RER) and ribosome-free smooth ER (SER) (Figure 1). The RER plays a key role in synthesis and transport of secretary/membrane proteins. The SER is critical for synthesis of lipids / sterols, storage and regulated release of calcium, and metabolism and detoxification. These peripheral ERs are highly dynamic and change their shapes and volumes on the demand of cellular needs (Federovitch et al., 2005; Park and Blackstone, 2010; Westrate et al., 2015).

ER dysfunction causes deleterious effects on the cells and is associated with many diseases including various neurological/neurodegenerative disorders. The major pathway involved in this is ER stress responses induced by disturbance of ER homeostasis due to protein misfolding and aggregation (Matus et al., 2011; Remondelli and Renna, 2017). In contrast, recent findings highlight another ER abnormalities associated with ER morphological alteration in neurological diseases. These are mainly caused by dysregulations of ER-resident membrane proteins. In this review, we discussed the ER dynamics and its derangement in neuropathogenesis.

\section{Peripheral ER Organization and Dynamics}

The peripheral ERs are morphologically subdivided into two domains, ER sheets and tubules (Figure 1). The sheets mainly located in perinuclear region and tend to be studded with ribosomes (RER), whereas tubules form cytoplasmic network and are largely devoid of ribosomes (SER). Different sets of proteins are involved in the organization of these distinct ER membrane domains (Figure 2; Park and Blackstone, 2010; Chen et al., 2013; Westrate et al., 2015). In the sheets, one of the key regulators is stubbed ribosome, which is elegantly shown by the experiment using two 
translation inhibitors, puromycin or cycloheximide; the ER sheets are disrupted by puromycin that quickly dissociates polysomes from ER membranes, but not by cycloheximide that stabilizes ribosomal association with translocons, in cultured cells (Puhka et al., 2007). Two transmembrane protein Climp63 and TMEM170A are also shown to be important regulators for RER sheet formation; knockdown of each one decreased ER sheets whereas its overexpression increased them (Shibata et al., 2010; Christodoulou et al., 2016). As for TMEM170A, its localization to nuclear envelopes and regulation of nuclear morphology are also reported (Christodoulou et al., 2016). Thus, the ER sheets are organized by different types of proteins (Figure 2).

On the contrary, the ER tubules are generated by a set of specific membrane proteins that contain one or two "hairpin" transmembrane domains. This domain is inserted

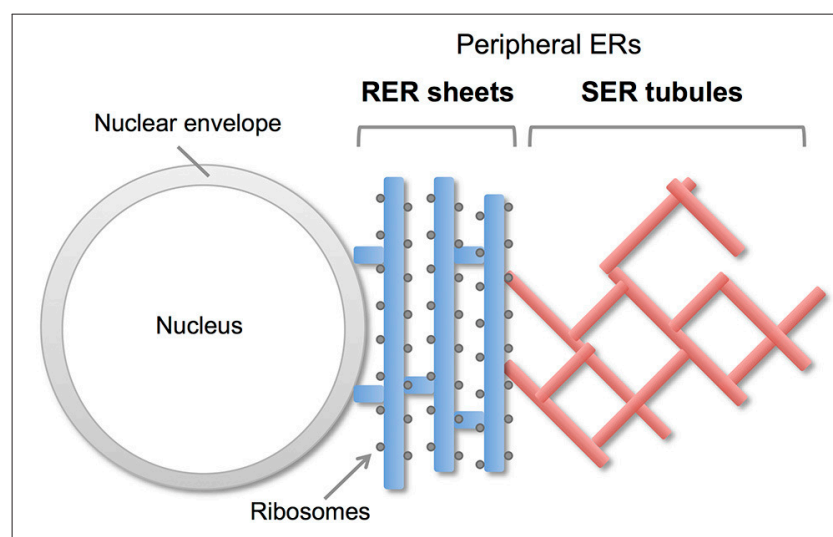

FIGURE 1 | Morphology of peripheral ERs in mammalian cells. The peripheral ERs are composed of two different subdomains; a ribosome-studded rough ER (RER) and a ribosome-free smooth ER (SER). The RERs form sheets in perinuclear region and play a role in protein synthesis and transport. The SERs form tubule networks in cytoplasm and are involved in lipids synthesis, calcium storage and detoxification. into outer leaflet of the membrane bilayer and suggested to generate curvature to bend the membrane (Figure 2; Park and Blackstone, 2010; Chen et al., 2013; Westrate et al., 2015). The membrane proteins include Reticulons (Rtn1-4), DP1 (deleted in polyposis locus 1; also known as REEP5), other REEPs (receptor expression-enhancing proteins; REEP1-4 and 6), Atlastin-1, M1Spastin and Lunapark. Among them, Reticulons, DP1 and Atlastin-1 are extensively analyzed and shown to be required for formation of ER tubular network in vitro (Hu et al., 2008; Wang et al., 2016; Powers et al., 2017) and in cultured cells (Voeltz et al., 2006; Hu et al., 2009). These three proteins interact with each other (Hu et al., 2009), and form immobile oligomer complex in the tubules (Shibata et al., 2008; Orso et al., 2009). Complex formation of REEP1 with Atlastin-1 and M1-Spastin and its contribution of ER tubule formation are also reported (Evans et al., 2006; Park et al., 2010). Thus, interconnected interactions of these membrane-inserting proteins mediate the ER tubule formation. Atlastin-1 and Lunapark also induce homotypic ER fusion to generate branched tubules (Orso et al., 2009; Chen et al., 2012, 2015). Binding to microtubule cytoskeleton through REEP1-4 and M1-spastin is further involved in establishment and maintenance of complicated tubular network (Park and Blackstone, 2010; Westrate et al., 2015).

\section{Disruption of ER Dynamics in Neurological Diseases}

Notably, some of the ER tubule proteins have been shown to be mutated in hereditary spastic paraplegia (HSP), a group of genetic disorders caused by a length-dependent, distal axonopathy of corticospinal motor neurons, leading to lower limb spasticity and weakness. Currently, over 40 different genetic loci (SPG1-45) are reported (Blackstone et al., 2011; Lo Giudice et al., 2014). Despite the large number of loci, about $60 \%$ of HSP patients harbor pathogenic mutations in one of three proteins: Spastin (SPG4), Atlastin-1 (SPG3A), or REEP1 (SPG31), all of which are the regulators of ER tubule formation as described

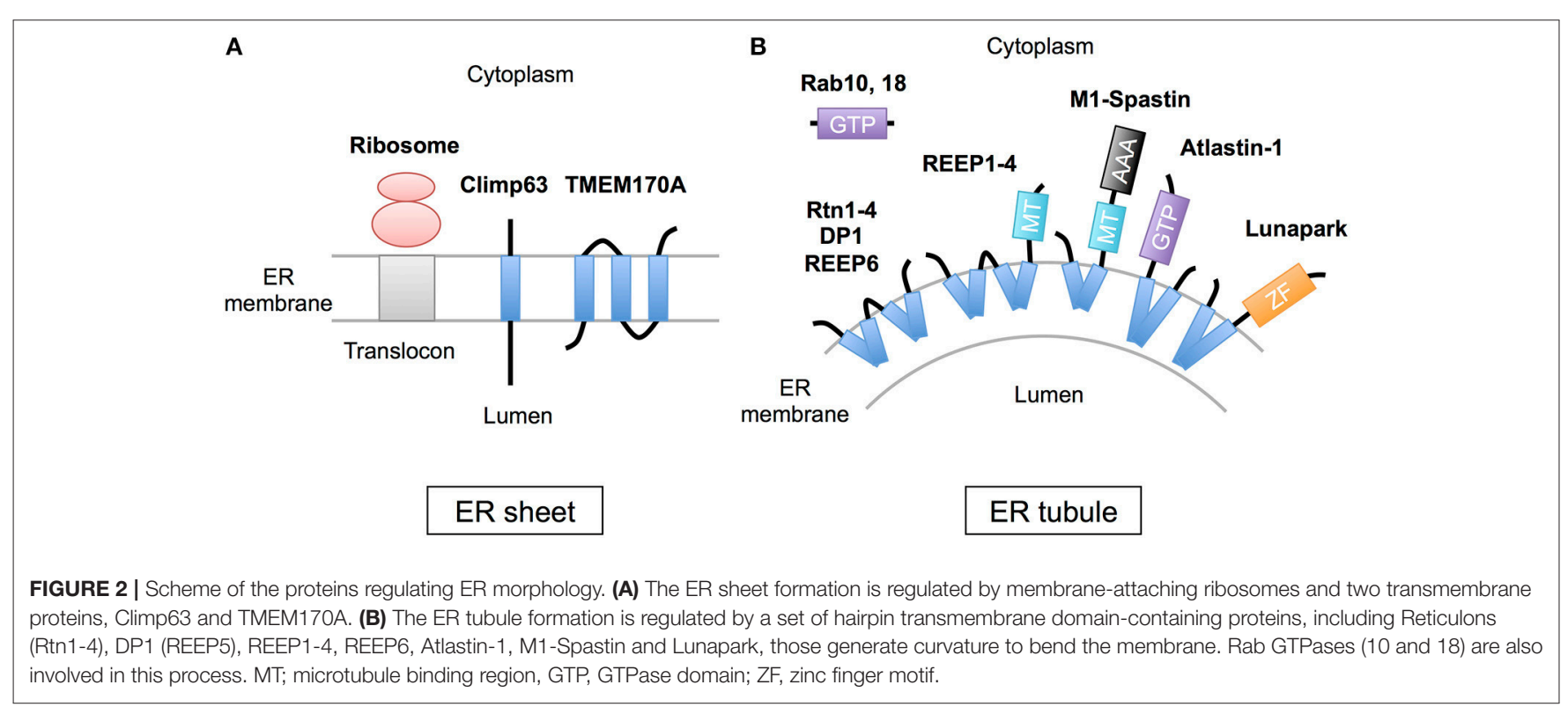


above. In addition, another ER tubule protein Rtn2 is also mutated in SPG12 patients. Thus, these SPG proteins are critical for motor neuron integrity in vivo. Indeed, knockdown of Spastin or Atlastin-1 in zebrafish induces axonal degeneration in motor neurons and locomotion defects (Butler et al., 2010; Fassier et al., 2010; Patten et al., 2014). In mice, mutation in Spastin or REEP1 causes progressive axonal degeneration of corticospinal motor neurons and motor defects (Tarrade et al., 2006; Beetz et al., 2013; Renvoise et al., 2016). Furthermore, peripheral ER complexity decreases in primary motor neurons of REEP1 mutant mice (Beetz et al., 2013). These observations suggest that these SPG proteins are necessary for axonal development and/or maintenance probably by modulating ER architecture in motor neurons. Because Spastin, Atlastin-1, and REEP1 are coenriched in axonal growth cones in cultured neurons (Park et al., 2010), they may regulate axonal growth by modulating ER tubule network dynamics.

The other factors involved in the ER tubule formation are Rab GTPases, central regulators of vesicle budding, motility, and fusion (Figure 2). Two Rab GTPases, Rab10, and Rab18, are shown to localize to ER tubules and its dysfunction results in ER tubule disorganization in cultured cells (English and Voeltz, 2013; Gerondopoulos et al., 2014). Notably, loss-of-function mutations of Rab18 or its regulator RabGAP cause Warburg Micro Syndrome, a rare autosomal recessive genetic disorder characterized by severe eye and brain abnormalities (Bem et al., 2011). Knockdown of Rab18 in zebrafish causes developmental abnormalities including microphthalmia and microcephaly (Bem et al., 2011). In addition, knockdown of Rab10 or Rab18 induces defects in neuronal differentiation in mouse brain cortex (Wang et al., 2011; Wu et al., 2016). These observations reveal critical roles of these Rab GTPases in neuronal development in vivo.

An ER membrane protein FAM134B is also linked to a neurological disease named hereditary sensory and autonomic neuropathy (HSAN) (Kurth et al., 2009). Interestingly, this protein is shown to be an autophagy receptor to mediate degradation of ER through autophagic system. This phenomenon is called ER-phagy, which is considered to be involved in ER homeostasis (Khaminets et al., 2015). Being its localization mainly to ER sheets, FAM134B is thought to be an ER sheetspecific autophagy receptor. Recently, an ER tubule protein Rtn3 is identified as another ER-phagy receptor, which induce fragmentation and autophagic degradation of ER tubules independently of FAM134B (Grumati et al., 2017). Thus, FAM134B and Rtn3 may regulate different ER membranes to regulate overall ER homeostasis.

\section{The ER Tubule Network and Neurodegenerative Diseases}

The ER tubules are interacting with other membranous organelles such as mitochondria, plasma membrane, peroxisomes and lysosomes. The mitochondria-associated ER membrane (MAM) mediates several fundamental cellular processes including calcium exchange, phospholipid exchange, intracellular trafficking and autophagy. The ER-mitochondria associations through MAM are shown to be disrupted in several neurodegenerative diseases such as Alzheimer's disease (AD), Parkinson's disease and amyotrophic lateral sclerosis with associated frontotemporal dementia (ALS/FTD), resulting in alteration in cellular functions regulated by MAM (Paillusson et al., 2016).

Direct implications of ER tubule proteins in disease pathogenesis have been reported. In superoxide dismutase 1 (SOD1) G93A transgenic mouse, a model for ALS, depletion of Rtn 4 accelerates disease onset and progression possibly by disrupting normal distribution of protein disulfide isomerase (PDI), suggesting protective role of Reticulon in motor neuron degeneration (Yang et al., 2009). As for AD, Rtn3 binds to and colocalizes with BACE1, a beta-secretase involved in amyloid precursor protein (APP) cleavage and amyloid beta production. Interestingly, overexpression of Rtn3 reduces the production of amyloid beta while its knockdown enhances it in cultured cells. Furthermore, Rtn3 blocks BACE1 interactions with APP, suggesting that the Reticulon negatively modulates BACE1 activity and amyloid beta production ( $\mathrm{He}$ et al., 2004). In addition, enhanced expression of Rtn3 suppresses amyloid plaque formation in transgenic mice expressing mutants for APP and presenilin-1 (Shi et al., 2009). In contrast to these beneficial effects, Rtn3 is found to be aggregated and accumulated in dystrophic neurites, named as Rtn3 immunoreactive dystrophic neurites (RIDNs) in brains of AD cases and mice brains expressing mutant APP. Furthermore, Rtn3 transgenic expression impairs spatial learning and memory as well as synaptic plasticity in mice, implying that RIDNs potentially contribute to $\mathrm{AD}$ cognitive dysfunction ( $\mathrm{Hu}$ et al., 2007). Thus, Rtn 3 may bidirectionally regulate $\mathrm{AD}$ pathogenesis in vivo.

\section{Biogenesis of Highly-Ordered ERs, the Stacked SERs}

In addition to the tubular architecture as described above, the SER is known to form highly-organized membranous structures where cisternae are stacked with ordered arrays (Federovitch et al., 2005; Borgese et al., 2006). This "stacked SER" is observed in the cells highly demanding SER-related functions such as lipid biosynthesis and drug metabolism. These include adrenal cells that produce large amount of sterol lipids, and liver cells of animal treated with phenobarbital (Feldman et al., 1981; Federovitch et al., 2005). Treatment of statins (cholesterol synthesis inhibitors) also leads to stacked SER formation by induced expression of an ER-resident enzyme, hydroxy-methylglutaryl (HMG)-CoA reductase (Singer et al., 1988; Borgese et al., 2006). Interestingly, overexpression of ER resident membrane proteins such as cytochrome b(5), P450, aldehyde dehydrogenase, Sec61 and Calnexin in cultured cells is shown to be sufficient for stacked SER formation (Yamamoto et al., 1996; Snapp et al., 2003; Korkhov and Zuber, 2009). Thus, load of ER-resident membrane proteins is one of the factors for stacked SER biogenesis.

As for the molecular mechanism, selective activation of ATF6 and following lipid synthesis are suggested to be involved in stacked SER generation upon cytochrome b(5) 


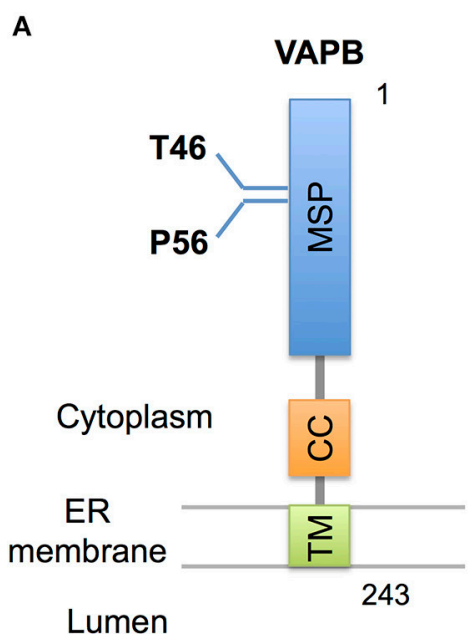

B

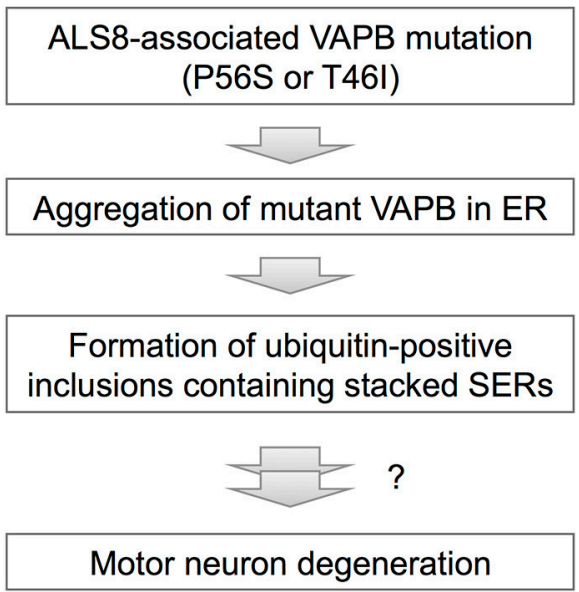

FIGURE 3 | VAPB mutations and SER pathology. (A) Domain structure of VAPB. VAPB is composed of the 125-residue major sperm protein (MSP), coiled coil (CC), and transmembrane (TM) domains. The residues Thr46 and Pro56 are mutated to lle and Ser, respectively, in familial ALS (ALS8). (B) A model for VAPB-pathology in ALS8. The mutant VAPB proteins are insolubilized and aggregated in ER, leading to formation of ubiquitin-positive inclusions containing stacked SER. The direct link of ER pathology to motor neuron degeneration is still uncertain, however.

expression (Maiuolo et al., 2011). Because of no inductions of ER chaperone expression, XBP1 splicing or eIF2-alpha phosphorylation, the usual unfolded protein response (UPR) pathway may not be involved in ATF6-dependent pathway. Knockdown of Syntaxin 18, a SNARE component involved in ER-Golgi transport and ER-network organization, also induces stacked SER formation (Iinuma et al., 2009). Furthermore, knockdown of a membrane protein Yip1A, which cycles between ER and early Golgi, induces formation of stacked SER (Dykstra et al., 2010). A compound phenyl-2-decanoyl-amino3-morpholino-1-propanol-hydrocholride (PDMP) that blocks membrane transport from ER to Golgi also induces generation of this type of ER (Sprocati et al., 2006). Experiments using several compounds further suggested that altering ionic homeostasis in ER is also an inducer of stacked SER formation independently of known UPR pathways (Varadarajan et al., 2012, 2013). Notably, the stacked SER formation often accompanies Golgi fragmentation and delay of ER export (Iinuma et al., 2009; Dykstra et al., 2010; Varadarajan et al., 2012). These observations suggest close relationship of ER-Golgi transport machineries, rather than canonical UPR pathways, to stacked SER biogenesis.

Despite the drastic alterations of ER and Golgi architectures, there is no report describing distinct reduction of cell viability. Importantly, the ER structure is reversible because depletion of the compounds such as PDMP leads to disappearance of stacked SER (Sprocati et al., 2006; Varadarajan et al., 2012). Thus, the stacked SER formation may not be the toxic inducer for cells but is suggested to be a novel stress response to cope with overload of ER membrane proteins independently of usual UPR pathways.

\section{Stacked SER Pathologies in Neurological Diseases}

Recent observations, however, indicate pathogenic significance of stacked SER in neurological diseases. In familial ALS
(ALS8), an ER-resident membrane protein, vesicle-associated membrane protein-associated protein B (VAPB) is mutated (Figure 3; Nishimura et al., 2004). Overexpressed mutant VAPB protein in cultured cells is insolubilized and aggregated in ER, leading to formation of ubiquitin-positive inclusions containing stacked SERs (Figure 3; Teuling et al., 2007; Fasana et al., 2010). In cultured neurons, the mutant VAPB overexpression induces Golgi fragmentation and cell death (Teuling et al., 2007). Furthermore, in VAPB mutant-transgenic mice ubiquitinpositive inclusions associated with stacked SER are developed in motor neurons (Tudor et al., 2010; Aliaga et al., 2013; Kuijpers et al., 2013; Qiu et al., 2013), and furthermore progressive loss of corticospinal motor neurons is observed in some of mice lines (Aliaga et al., 2013). Although the neurotoxic effect of mutant VAPB is still controversial because other transgenic mice lines do not show neuronal loss or motor phenotypes (Tudor et al., 2010; Kuijpers et al., 2013; Qiu et al., 2013), VAPB mutant knock-in mice are shown to display slow progression of motor behavior defects (Larroquette et al., 2015), suggesting a certain involvement of VAPB mutation on motor neuron dysfunction.

Torsion dystonia-1 (DYT1) is the most common inherited dystonia characterized by involuntary muscle contractions and abnormal postures, which is caused by mutation in TorsinA, an ER glycoprotein belonging to AAA family of proteins (Ozelius et al., 1997). The mutant TorsinA forms cytoplasmic inclusions containing stacked SER in cultured neuronal cells (Hewett et al., 2000; Gonzalez-Alegre and Paulson, 2004). In peripheral nervous system, mutation in peripheral myelin protein 22 (PMP22) is linked to Charcot-Marie-Tooth disease, a sensorineural peripheral polyneuropathy. PMP22 is expressed in Schwann cells and its mutation induces formation of cytoplasmic inclusion containing mutant protein in association with stacked SER formation (Dickson et al., 2002). These observations further provide the pathological significance of cytoplasmic inclusions containing stacked SER in neurological diseases. 


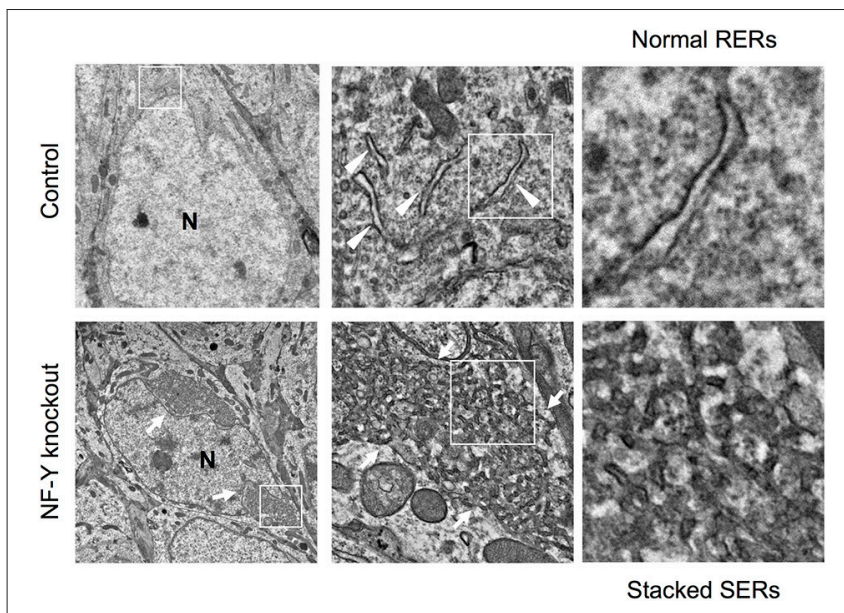

FIGURE 4 | SER pathology in NF-Y knockout neurons. Electron microgram of hippocampal CA1 neurons in control and NF-Y knockout mouse. Boxed areas are enlarged in the next panels. The neurons of NF-Y knockout mouse contain stacked SERs at perinuclear regions (arrows in lower panels) whereas control mouse neurons contain normal RERs (arrowheads in upper panels). $\mathrm{N}$ indicates nucleus. The data are reproduced from our previous paper (Yamanaka et al., 2014) with slight modifications.

A novel mouse model for stacked SER pathology in brain neurons was recently established (Yamanaka et al., 2014, 2016). Importantly, it does not involve disease-associated gene mutation but just caused by inactivation of CCAAT-binding factor NF-Y, a ubiquitous transcription factor shown to be affected in polyglutamine diseases (Yamanaka et al., 2008; Katsuno et al., 2010; Huang et al., 2011). The neuron-specific knockdown of NF-Y induces insolubilization of various membrane proteins including Calnexin, Reticulon, Atlastin-1, APP and Carboxypeptidase E together with ubiquitin and p62/Sqstm1, all of which are accumulated on ER (Yamanaka et al., 2014, 2016). It also accompanies perinuclear accumulation of ribosome-free SERs and Golgi disassembly (Figure 4). Chromatin immunoprecipitation identifies several genes involved in protein folding in ER and ER-associated degradation

\section{REFERENCES}

Aliaga, L., Lai, C., Yu, J., Chub, N., Shim, H., Sun, L., et al. (2013). Amyotrophic lateral sclerosis-related VAPB P56S mutation differentially affects the function and survival of corticospinal and spinal motor neurons. Hum. Mol. Genet. 22, 4293-4305. doi: 10.1093/hmg/ddt279

Beetz, C., Koch, N., Khundadze, M., Zimmer, G., Nietzsche, S., Hertel, N., et al. (2013). A spastic paraplegia mouse model reveals REEP1dependent ER shaping. J. Clin. Invest. 123, 4273-4282. doi: 10.1172/ JCI65665

Bem, D., Yoshimura, S., Nunes-Bastos, R., Bond, F. C., Kurian, M. A., Rahman, F., et al. (2011). Loss-of-function mutations in RAB18 cause Warburg micro syndrome. Am. J. Hum. Genet. 88, 499-507. doi: 10.1016/j.ajhg.2011.03.012

Blackstone, C., O’kane, C. J., and Reid, E. (2011). Hereditary spastic paraplegias: membrane traffic and the motor pathway. Nat. Rev. Neurosci. 12, 31-42. doi: $10.1038 / \mathrm{nrn} 2946$
(ERAD) as targets of NF-Y, suggesting a critical role of NF-Y in ER protein homeostasis to maintain normal ER architecture in brain neurons.

\section{CONCLUSIONS}

Among the peripheral ERs, SERs appear to be deranged in several neurological diseases through at least two pathways. One is disturbance of peripheral ER network in the diseases such as HSPs, which is caused by mutations of genes required for SER tubule organization. The SER network disruption may also be involved in Alzheimer's disease and ALS pathogenesis. Another one is development of highly-ordered stacked SER in the diseases such as ALS8, which is caused by overload of misfolded and insolubilized proteins on ER membranes. NF-Ymediated gene regulatory pathway can be involved in this atypical ER pathogenesis. Finding the way to restore SER networks is a future challenging issue toward the cure of these ER-related neurological disorders.

\section{AUTHOR CONTRIBUTIONS}

All authors listed have made a substantial, direct and intellectual contribution to the work, and approved it for publication.

\section{ACKNOWLEDGMENTS}

This work was supported by Grant-in-Aid for Scientific Research on Innovative Areas (Brain Protein Aging and Dementia Control) (15H01567) from Ministry of Education, Culture, Sports, Science and Technology (MEXT), and Grant-inAids from MEXT/The Japan Society for the Promotion of Science (JSPS). (17H01564, 16H01345, 17KT0131, 15K06762), Takeda Science Foundation, and Center for Baby Science in Doshisha University (Joint Usage/Research Center accredited by MEXT). This work is partially supported by the Strategic Research Program for Brain Sciences from Japan Agency for Medical Research and Development, AMED.
Borgese, N., Francolini, M., and Snapp, E. (2006). Endoplasmic reticulum architecture: structures in flux. Curr. Opin. Cell Biol. 18, 358-364. doi: 10.1016/j.ceb.2006.06.008

Butler, R., Wood, J. D., Landers, J. A., and Cunliffe, V. T. (2010). Genetic and chemical modulation of spastin-dependent axon outgrowth in zebrafish embryos indicates a role for impaired microtubule dynamics in hereditary spastic paraplegia. Dis. Model. Mech. 3, 743-751. doi: 10.1242/dmm.004002

Chen, S., Desai, T., McNew, J. A., Gerard, P., Novick, P. J., and FerroNovick, S. (2015). Lunapark stabilizes nascent three-way junctions in the endoplasmic reticulum. Proc. Natl. Acad. Sci. U.S.A. 112, 418-423. doi: $10.1073 /$ pnas. 1423026112

Chen, S., Novick, P., and Ferro-Novick, S. (2012). ER network formation requires a balance of the dynamin-like GTPase Seylp and the Lunapark family member Lnp1p. Nat. Cell Biol. 14, 707-716. doi: 10.1038/ncb2523

Chen, S., Novick, P., and Ferro-Novick, S. (2013). ER structure and function. Curr. Opin. Cell Biol. 25, 428-433. doi: 10.1016/j.ceb.2013.02.006 
Christodoulou, A., Santarella-Mellwig, R., Santama, N., and Mattaj, I. W. (2016). Transmembrane protein TMEM170A is a newly discovered regulator of ER and nuclear envelope morphogenesis in human cells. J. Cell Sci. 129, 1552-1565. doi: $10.1242 /$ jcs. 175273

Dickson, K. M., Bergeron, J. J., Shames, I., Colby, J., Nguyen, D. T., Chevet, E., et al. (2002). Association of calnexin with mutant peripheral myelin protein-22 ex vivo: a basis for "gain-of-function" ER diseases. Proc. Natl. Acad. Sci. U.S.A. 99, 9852-9857. doi: 10.1073/pnas.152621799

Dykstra, K. M., Pokusa, J. E., Suhan, J., and Lee, T. H. (2010). Yip1A structures the mammalian endoplasmic reticulum. Mol. Biol. Cell 21, 1556-1568. doi: 10.1091/mbc.E09-12-1002

English, A. R., and Voeltz, G. K. (2013). Rab10 GTPase regulates ER dynamics and morphology. Nat. Cell Biol. 15, 169-178. doi: 10.1038/ncb2647

Evans, K., Keller, C., Pavur, K., Glasgow, K., Conn, B., and Lauring, B. (2006). Interaction of two hereditary spastic paraplegia gene products, spastin and atlastin, suggests a common pathway for axonal maintenance. Proc. Natl. Acad. Sci. U.S.A. 103, 10666-10671. doi: 10.1073/pnas. 0510863103

Fasana, E., Fossati, M., Ruggiano, A., Brambillasca, S., Hoogenraad, C. C., Navone, F., et al. (2010). A VAPB mutant linked to amyotrophic lateral sclerosis generates a novel form of organized smooth endoplasmic reticulum. FASEB J. 24, 1419-1430. doi: 10.1096/fj.09-147850

Fassier, C., Hutt, J. A., Scholpp, S., Lumsden, A., Giros, B., Nothias, F., et al. (2010). Zebrafish atlastin controls motility and spinal motor axon architecture via inhibition of the BMP pathway. Nat. Neurosci. 13, 1380-1387. doi: $10.1038 / \mathrm{nn} .2662$

Federovitch, C. M., Ron, D., and Hampton, R. Y. (2005). The dynamic ER: experimental approaches and current questions. Curr. Opin. Cell Biol. 17, 409-414. doi: 10.1016/j.ceb.2005.06.010

Feldman, D., Swarm, R. L., and Becker, J. (1981). Ultrastructural study of rat liver and liver neoplasms after long-term treatment with phenobarbital. Cancer Res. $41,2151-2162$

Gerondopoulos, A., Bastos, R. N., Yoshimura, S., Anderson, R., Carpanini, S., Aligianis, I., et al. (2014). Rab18 and a Rab18 GEF complex are required for normal ER structure. J. Cell Biol. 205, 707-720. doi: 10.1083/jcb.201403026

Gonzalez-Alegre, P., and Paulson, H. L. (2004). Aberrant cellular behavior of mutant torsin A implicates nuclear envelope dysfunction in DYT1 dystonia. J. Neurosci. 24, 2593-2601. doi: 10.1523/JNEUROSCI.4461-03.2004

Grumati, P., Morozzi, G., Holper, S., Mari, M., Harwardt, M. I., Yan, R., et al. (2017). Full length RTN3 regulates turnover of tubular endoplasmic reticulum via selective autophagy. Elife 6:e25555 doi: 10.7554/eLife.25555

He, W., Lu, Y., Qahwash, I., Hu, X. Y., Chang, A., and Yan, R. (2004). Reticulon family members modulate BACE1 activity and amyloid-beta peptide generation. Nat. Med. 10, 959-965. doi: 10.1038/nm1088

Hewett, J., Gonzalez-Agosti, C., Slater, D., Ziefer, P., Li, S., Bergeron, D., et al. (2000). Mutant torsinA, responsible for early-onset torsion dystonia, forms membrane inclusions in cultured neural cells. Hum. Mol. Genet. 9, 1403-1413. doi: $10.1093 / \mathrm{hmg} / 9.9 .1403$

Hu, J., Shibata, Y., Voss, C., Shemesh, T., Li, Z., Coughlin, M., et al. (2008). Membrane proteins of the endoplasmic reticulum induce high-curvature tubules. Science 319, 1247-1250. doi: 10.1126/science.1153634

Hu, J., Shibata, Y., Zhu, P. P., Voss, C., Rismanchi, N., Prinz, W. A., et al. (2009). A class of dynamin-like GTPases involved in the generation of the tubular ER network. Cell 138, 549-561. doi: 10.1016/j.cell.2009.05.025

Hu, X., Shi, Q., Zhou, X., He, W., Yi, H., Yin, X., et al. (2007). Transgenic mice overexpressing reticulon 3 develop neuritic abnormalities. EMBO J. 26, 2755-2767. doi: 10.1038/sj.emboj.7601707

Huang, S., Ling, J. J., Yang, S., Li, X. J., and Li, S. (2011). Neuronal expression of TATA box-binding protein containing expanded polyglutamine in knock-in mice reduces chaperone protein response by impairing the function of nuclear factor-Y transcription factor. Brain 134, 1943-1958. doi: 10.1093/brain/awr146

Iinuma, T., Aoki, T., Arasaki, K., Hirose, H., Yamamoto, A., Samata, R., et al. (2009). Role of syntaxin 18 in the organization of endoplasmic reticulum subdomains. J. Cell Sci. 122, 1680-1690. doi: 10.1242/jcs.036103

Katsuno, M., Adachi, H., Minamiyama, M., Waza, M., Doi, H., Kondo, N., et al. (2010). Disrupted transforming growth factor-beta signaling in spinal and bulbar muscular atrophy. J. Neurosci. 30, 5702-5712. doi: 10.1523/JNEUROSCI.0388-10.2010
Khaminets, A., Heinrich, T., Mari, M., Grumati, P., Huebner, A. K., Akutsu, M., et al. (2015). Regulation of endoplasmic reticulum turnover by selective autophagy. Nature 522, 354-358. doi: 10.1038/nature14498

Korkhov, V. M., and Zuber, B. (2009). Direct observation of molecular arrays in the organized smooth endoplasmic reticulum. BMC Cell Biol. 10:59. doi: 10.1186/1471-2121-10-59

Kuijpers, M., Van Dis, V., Haasdijk, E. D., Harterink, M., Vocking, K., Post, J. A., et al. (2013). Amyotrophic lateral sclerosis (ALS)-associated VAPB-P56S inclusions represent an ER quality control compartment. Acta Neuropathol. Commun. 1:24. doi: 10.1186/2051-5960-1-24

Kurth, I., Pamminger, T., Hennings, J. C., Soehendra, D., Huebner, A. K., Rotthier, A., et al. (2009). Mutations in FAM134B, encoding a newly identified Golgi protein, cause severe sensory and autonomic neuropathy. Nat. Genet. 41, 1179-1181. doi: 10.1038/ng.464

Larroquette, F., Seto, L., Gaub, P. L., Kamal, B., Wallis, D., Lariviere, R., et al. (2015). Vapb/Amyotrophic lateral sclerosis 8 knock-in mice display slowly progressive motor behavior defects accompanying ER stress and autophagic response. Hum. Mol. Genet. 24, 6515-6529. doi: 10.1093/hmg/ddv360

Lo Giudice, T., Lombardi, F., Santorelli, F. M., Kawarai, T., and Orlacchio, A. (2014). Hereditary spastic paraplegia: clinical-genetic characteristics and evolving molecular mechanisms. Exp. Neurol. 261, 518-539. doi: 10.1016/j.expneurol.2014.06.011

Maiuolo, J., Bulotta, S., Verderio, C., Benfante, R., and Borgese, N. (2011). Selective activation of the transcription factor ATF6 mediates endoplasmic reticulum proliferation triggered by a membrane protein. Proc. Natl. Acad. Sci. U.S.A. 108, 7832-7837. doi: 10.1073/pnas.1101379108

Matus, S., Glimcher, L. H., and Hetz, C. (2011). Protein folding stress in neurodegenerative diseases: a glimpse into the ER. Curr. Opin. Cell Biol. 23, 239-252. doi: 10.1016/j.ceb.2011.01.003

Nishimura, A. L., Mitne-Neto, M., Silva, H. C., Richieri-Costa, A., Middleton, S., Cascio, D., et al. (2004). A mutation in the vesicle-trafficking protein VAPB causes late-onset spinal muscular atrophy and amyotrophic lateral sclerosis. Am. J. Hum. Genet. 75, 822-831. doi: 10.1086/425287

Orso, G., Pendin, D., Liu, S., Tosetto, J., Moss, T. J., Faust, J. E., et al. (2009). Homotypic fusion of ER membranes requires the dynamin-like GTPase atlastin. Nature 460, 978-983. doi: 10.1038/nature08280

Ozelius, L. J., Hewett, J. W., Page, C. E., Bressman, S. B., Kramer, P. L., Shalish, C., et al. (1997). The early-onset torsion dystonia gene (DYT1) encodes an ATP-binding protein. Nat. Genet. 17, 40-48. doi: 10.1038/ng0997-40

Paillusson, S., Stoica, R., Gomez-Suaga, P., Lau, D. H., Mueller, S., Miller, T., et al. (2016). There's something wrong with my MAM; the ERMitochondria axis and neurodegenerative diseases. Trends Neurosci. 39, 146-157. doi: 10.1016/j.tins.2016.01.008

Park, S. H., and Blackstone, C. (2010). Further assembly required: construction and dynamics of the endoplasmic reticulum network. EMBO Rep. 11, 515-521. doi: 10.1038/embor.2010.92

Park, S. H., Zhu, P. P., Parker, R. L., and Blackstone, C. (2010). Hereditary spastic paraplegia proteins REEP1, spastin, and atlastin-1 coordinate microtubule interactions with the tubular ER network. J. Clin. Invest. 120, 1097-1110. doi: 10.1172/JCI40979

Patten, S. A., Armstrong, G. A., Lissouba, A., Kabashi, E., Parker, J. A., and Drapeau, P. (2014). Fishing for causes and cures of motor neuron disorders. Dis. Model. Mech. 7, 799-809. doi: 10.1242/dmm.015719

Powers, R. E., Wang, S., Liu, T. Y., and Rapoport, T. A. (2017). Reconstitution of the tubular endoplasmic reticulum network with purified components. Nature 543, 257-260. doi: 10.1038/nature21387

Puhka, M., Vihinen, H., Joensuu, M., and Jokitalo, E. (2007). Endoplasmic reticulum remains continuous and undergoes sheet-to-tubule transformation during cell division in mammalian cells. J. Cell Biol. 179, 895-909. doi: $10.1083 /$ jcb.200705112

Qiu, L., Qiao, T., Beers, M., Tan, W., Wang, H., Yang, B., et al. (2013). Widespread aggregation of mutant VAPB associated with ALS does not cause motor neuron degeneration or modulate mutant SOD1 aggregation and toxicity in mice. Mol. Neurodegener. 8:1. doi: 10.1186/1750-1326-8-1

Remondelli, P., and Renna, M. (2017). The Endoplasmic Reticulum unfolded protein response in neurodegenerative disorders and its potential therapeutic significance. Front. Mol. Neurosci. 10:187. doi: 10.3389/fnmol.2017.00187 
Renvoise, B., Malone, B., Falgairolle, M., Munasinghe, J., Stadler, J., Sibilla, C., et al. (2016). Reep1 null mice reveal a converging role for hereditary spastic paraplegia proteins in lipid droplet regulation. Hum. Mol. Genet. 25, 5111-5125. doi: 10.1093/hmg/ddw315.

Shi, Q., Prior, M., He, W., Tang, X., Hu, X., and Yan, R. (2009), Reduced amyloid deposition in mice overexpressing RTN3 is adversely affected by preformed dystrophic neurites. J. Neurosci. 29, 9163-9173. doi: 10.1523/JNEUROSCI.5741-08.2009

Shibata, Y., Shemesh, T., Prinz, W. A., Palazzo, A. F., Kozlov, M. M., and Rapoport, T. A. (2010). Mechanisms determining the morphology of the peripheral ER. Cell 143, 774-788. doi: 10.1016/j.cell.2010.11.007

Shibata, Y., Voss, C., Rist, J. M., Hu, J., Rapoport, T. A., Prinz, W. A., et al. (2008). The reticulon and DP1/Yoplp proteins form immobile oligomers in the tubular endoplasmic reticulum. J. Biol. Chem. 283, 18892-18904. doi: 10.1074/jbc.M800986200

Singer, I.i, Scott, S., Kazazis, D. M., and Huff, J. W. (1988). Lovastatin, an inhibitor of cholesterol synthesis, induces hydroxymethylglutaryl-coenzyme a reductase directly on membranes of expanded smooth endoplasmic reticulum in rat hepatocytes. Proc. Natl. Acad. Sci. U.S.A. 85, 5264-5268. doi: $10.1073 /$ pnas.85.14.5264

Snapp, E. L., Hegde, R. S., Francolini, M., Lombardo, F., Colombo, S., Pedrazzini, E., et al. (2003). Formation of stacked ER cisternae by low affinity protein interactions. J. Cell Biol. 163, 257-269. doi: 10.1083/jcb.200306020

Sprocati, T., Ronchi, P., Raimondi, A., Francolini, M., and Borgese, N. (2006). Dynamic and reversible restructuring of the ER induced by PDMP in cultured cells. J. Cell Sci. 119, 3249-3260. doi: 10.1242/jcs.03058

Tarrade, A., Fassier, C., Courageot, S., Charvin, D., Vitte, J., Peris, L., et al. (2006). A mutation of spastin is responsible for swellings and impairment of transport in a region of axon characterized by changes in microtubule composition. Hum. Mol. Genet. 15, 3544-3558. doi: 10.1093/hmg/ddl431

Teuling, E., Ahmed, S., Haasdijk, E., Demmers, J., Steinmetz, M. O., Akhmanova, A., et al. (2007). Motor neuron disease-associated mutant vesicle-associated membrane protein-associated protein (VAP) B recruits wild-type VAPs into endoplasmic reticulum-derived tubular aggregates. J. Neurosci. 27, 9801-9815. doi: 10.1523/JNEUROSCI.2661-07.2007

Tudor, E. L., Galtrey, C. M., Perkinton, M. S., Lau, K. F., De Vos, K. J., Mitchell, J. C., et al. (2010). Amyotrophic lateral sclerosis mutant vesicleassociated membrane protein-associated protein-B transgenic mice develop TAR-DNA-binding protein-43 pathology. Neuroscience 167, 774-785. doi: 10.1016/j.neuroscience.2010.02.035

Varadarajan, S., Bampton, E. T., Smalley, J. L., Tanaka, K., Caves, R. E., Butterworth, M., et al. (2012). A novel cellular stress response characterised by a rapid reorganisation of membranes of the endoplasmic reticulum. Cell Death Differ. 19, 1896-1907. doi: 10.1038/cdd.2012.108

Varadarajan, S., Tanaka, K., Smalley, J. L., Bampton, E. T., Pellecchia, M., Dinsdale, D., et al. (2013). Endoplasmic reticulum membrane reorganization is regulated by ionic homeostasis. PLoS ONE 8:e56603. doi: 10.1371/journal.pone.0056603
Voeltz, G. K., Prinz, W. A., Shibata, Y., Rist, J. M., and Rapoport, T. A. (2006). A class of membrane proteins shaping the tubular endoplasmic reticulum. Cell 124, 573-586. doi: 10.1016/j.cell.2005.11.047

Wang, S., Tukachinsky, H., Romano, F. B., and Rapoport, T. A. (2016). Cooperation of the ER-shaping proteins atlastin, lunapark, and reticulons to generate a tubular membrane network. Elife 5:e18605 doi: 10.7554/eLife.18605

Wang, T., Liu, Y., Xu, X. H., Deng, C. Y., Wu, K. Y., Zhu, J., et al. (2011) Lgl1 activation of rab10 promotes axonal membrane trafficking underlying neuronal polarization. Dev. Cell 21, 431-444. doi: 10.1016/j.devcel.2011. 07.007

Westrate, L. M., Lee, J. E., Prinz, W. A., and Voeltz, G. K. (2015). Form follows function: the importance of endoplasmic reticulum shape. Annu. Rev. Biochem. 84, 791-811. doi: 10.1146/annurev-biochem-072711-163501

Wu, Q., Sun, X., Yue, W., Lu, T., Ruan, Y., Chen, T., et al. (2016). RAB18, a protein associated with Warburg Micro syndrome, controls neuronal migration in the developing cerebral cortex. Mol. Brain 9:19. doi: 10.1186/s13041-016-0198-2

Yamamoto, A., Masaki, R., and Tashiro, Y. (1996). Formation of crystalloid endoplasmic reticulum in COS cells upon overexpression of microsomal aldehyde dehydrogenase by cDNA transfection. J. Cell Sci. 109(Pt 7), 1727-1738.

Yamanaka, T., Miyazaki, H., Oyama, F., Kurosawa, M., Washizu, C., Doi, H., et al. (2008). Mutant Huntingtin reduces HSP70 expression through the sequestration of NF-Y transcription factor. EMBO J. 27, 827-839. doi: 10.1038/emboj.2008.23

Yamanaka, T., Tosaki, A., Kurosawa, M., Matsumoto, G., Koike, M., Uchiyama, Y., et al. (2014). NF-Y inactivation causes atypical neurodegeneration characterized by ubiquitin and p62 accumulation and endoplasmic reticulum disorganization. Nat. Commun. 5:3354. doi: 10.1038/ncomms4354

Yamanaka, T., Tosaki, A., Miyazaki, H., Kurosawa, M., Koike, M., Uchiyama, Y., et al. (2016). Differential roles of NF-Y transcription factor in ER chaperone expression and neuronal maintenance in the CNS. Sci. Rep. 6:34575. doi: 10.1038/srep34575

Yang, Y. S., Harel, N. Y., and Strittmatter, S. M. (2009). Reticulon-4A (NogoA) redistributes protein disulfide isomerase to protect mice from SOD1dependent amyotrophic lateral sclerosis. J. Neurosci. 29, 13850-13859. doi: 10.1523/JNEUROSCI.2312-09.2009

Conflict of Interest Statement: The authors declare that the research was conducted in the absence of any commercial or financial relationships that could be construed as a potential conflict of interest.

Copyright (C) 2018 Yamanaka and Nukina. This is an open-access article distributed under the terms of the Creative Commons Attribution License (CC BY). The use, distribution or reproduction in other forums is permitted, provided the original author(s) and the copyright owner are credited and that the original publication in this journal is cited, in accordance with accepted academic practice. No use, distribution or reproduction is permitted which does not comply with these terms. 